\title{
Soft Fixed Point Theorems for the Soft Comparable Contractions
}

\author{
Chi-Ming Chen $\mathbb{D}^{1},{ }^{1}$ Zhi-Hao Xu ${ }^{\mathbb{D}}{ }^{1}$, and Erdal Karapinar $\mathbb{D}^{2,3,4}$ \\ ${ }^{1}$ Institute for Computational and Modeling Science, National Tsing Hua University, Taiwan \\ ${ }^{2}$ Division of Applied Mathematics, Thu Dau Mot University, Binh Duong Province, Vietnam \\ ${ }^{3}$ Department of Medical Research, CMU Hospital, China Medical University, 40402 Taichung, Taiwan \\ ${ }^{4}$ Department of Mathematics, Cankaya University, 06790 Etimesgut, Ankara, Turkey
}

Correspondence should be addressed to Chi-Ming Chen; ming@mail.nd.nthu.edu.tw and Erdal Karapinar; erdalkarapinar@tdmu.edu.vn

Received 13 January 2021; Revised 29 January 2021; Accepted 3 February 2021; Published 11 March 2021

Academic Editor: Tuncer Acar

Copyright (C) 2021 Chi-Ming Chen et al. This is an open access article distributed under the Creative Commons Attribution License, which permits unrestricted use, distribution, and reproduction in any medium, provided the original work is properly cited.

\begin{abstract}
In this article, we introduce the notions of a soft inf-comparable contraction and soft comparable Meir-Keeler contraction in a soft metric space. Furthermore, we prove two soft fixed point theorems which assure the existence of soft fixed points for these two types of comparable contractions. The obtained results not only generalize but also unify many recent fixed point results in the literature.
\end{abstract}

\section{Introduction and Preliminaries}

It is the main feature of mathematical study to produce different methods and tools to perceive the behavior of systems that we have difficulty understanding with known methods. In particular, it may be necessary to deal with systems that contain uncertainties and to use inaccurate data in different situations. With this motivation, one of the mathematical tools used to deal with the necessities of systems established with uncertainty and to analyze the models created by the uncertainties and uncertainties already existing in the data is the Fuzzy Set Theory. Fuzzy sets were introduced by Zadeh [1] for dealing with the uncertainties on its own limits. Another mathematical tool to deal with the uncertainties is the soft set that was introduced by Molodtsov [2]. In this paper, we shall focus on the soft set theory. The topology based on the soft sets was defined by Cagman et al. [3]. They also considered the basic topological notions over soft sets. On the other hand, a soft real set and soft real number were proposed successfully by Das and Samanta [4]. Furthermore, the same authors in considered the notions of a soft metric and its topology, properly. After then, Abbas et al. [5] proved a fixed point theorem by introducing the notion of soft contraction mapping over the soft metric space. Application potential of the soft sets in various distinct research topics is very rich and wide, for example, the smoothness of func- tions, game theory, operation research, probability theory, and measurement theory. For more details on soft sets and application, we can refer to, e.g., [3, 4, 6-12].

As usual, $\mathbb{R}$ denotes real numbers and $\mathbb{R}^{+}:=[0, \infty)$. Furthermore, the letters $\mathbb{Z}, \mathbb{N}$ denote integers and natural numbers, respectively. The symbol $B(\mathbb{R})$ denotes the collection of all nonempty bounded subsets of $\mathbb{R}$.

We shall denote an initial universe $\Omega$. We set $\mathscr{P}$ as a set of parameters. As usual, $2^{\Omega}$ denotes the collection of all subsets of $\Omega$. For a nonempty subset $S$ of $\mathscr{P}$, we consider a set-valued mapping $T: S \rightarrow 2^{\Omega}$ for all $\tau \notin A$ with $T(\tau)=\phi$. We define a pair $(T, A)$ on $\Omega$ as

$$
(T, S)=\{(T(\tau), \tau): \tau \in \mathscr{P}\}
$$

Here, $(T, S)$ is called a soft set [2]. The symbol $\delta(\Omega)$ represents the collection of all soft sets on $\Omega$.

A soft set $(T, S)$ on $\Omega$ is called null soft [11] (respectively, absolute soft set [11]) represented by, respectively, $\tilde{S}$ if $T(\tau)=$ (respectively, $T(\tau)=S$ ) for all $\tau \in S$. We presume that $\left(T_{1}, S_{1}\right)$ and $\left(T_{2}, S_{2}\right)$ are two soft sets on $\Omega$. We define the intersection [11] of the mentioned two sets above as a soft set $\left(T_{3}, S_{3}\right)$, denoted by $\left(T_{1}, S_{1}\right) \cap \sim\left(T_{2}, S_{2}\right)=\left(T_{3}, S_{3}\right)$, where $S_{3}=S_{1} \cap S_{2}$, and for each $\tau \in S_{3}, T_{3}(\tau)=T_{1}(\tau) \cap T_{2}(\tau)$. As expected, we define the union of $\left(T_{1}, S_{1}\right)$ and $\left(T_{2}, S_{2}\right)$ [11] as a soft set 
$\left(T_{3}, S_{3}\right)$, denoted by $\left(T_{1}, A\right) \cup\left(T_{2}, B\right)=\left(T_{3}, C\right)$ where $S_{3}=$ $S_{1} \cup S_{2}$ and for each $\tau \in S_{3}$,

$$
T_{3}(\tau)= \begin{cases}T_{1}(\tau), & \text { if } \tau \in S_{1} \backslash S_{2}, \\ T_{2}(\tau), & \text { if } \tau \in S_{2} \backslash S_{1}, \\ T_{1}(\tau) \cup T_{2}(\tau), & \text { if } \tau \in S_{1} \cap S_{2}\end{cases}
$$

We use the notation $\left(T^{c}, \mathscr{P}\right)$ to indicate the complement [11] of soft set $(T, \mathscr{P})$ on $\Omega$ where $T^{c}: X \rightarrow 2^{\Omega}$ is a mapping given by $T^{\mathcal{C}}(\tau)=\Omega \backslash T(\tau)$ for all $\tau \in \mathscr{P}$.

A mapping $T: \mathscr{P} \rightarrow B(\mathbb{R})$ is called a soft real set [13]. The symbol $\mathbb{R}^{+}(\mathscr{P})$ is used to denote the set of all nonnegative soft real numbers. If $(T, \mathscr{P})$ is a singleton soft set, then it is called a soft real number. Regarding the corresponding soft set, soft real numbers will be denoted as $\tilde{\gamma}, \tilde{\eta}, \tilde{\xi}$, etc. In particular, $\overline{0}$ and $\tilde{1}$ are the soft real numbers where $\overline{0}(\tau)=0, \tilde{1}(\tau)$ $=1$ for all $\tau \in \mathscr{P}$.

For two soft real numbers, for all $\tau \in \mathscr{P}$, we have the following inequalities [13]:

(1) $\gamma^{\sim} \leq \sim \tilde{\eta}$ if $\gamma^{\sim}(\tau) \leq \sim \tilde{\eta}(\tau)$

(2) $\gamma^{\sim} \geq \sim \tilde{\eta}$ if $\gamma^{\sim}(\tau) \geq \sim \tilde{\eta}(\tau)$

(3) $\gamma^{\sim}<\sim \tilde{\eta}$ if $\gamma^{\sim}(\tau)<\sim \tilde{\eta}(\tau)$

(4) $\gamma^{\sim}>\sim \tilde{\eta}$ if $\gamma^{\sim}(\tau)>\sim \tilde{\eta}(\tau)$

\section{Definition 1.}

(1) The mapping $\phi: \mathbb{R}^{+}(\mathscr{P}) \rightarrow \mathbb{R}^{+}(\mathscr{P})$ is called soft increasing, if

$$
r^{\sim}<\tilde{t} \Rightarrow \phi\left(r^{\sim}\right)<\sim \phi(\tilde{t}) .
$$

(2) The mapping $\phi: \mathbb{R}^{+}(\mathscr{P}) \rightarrow \mathbb{R}^{+}(\mathscr{P})$ is called soft continuous at $a^{\sim} \in \sim \mathbb{R}^{+}(\mathscr{P})$, if for every $\gamma^{\sim}>\overline{0}$, there exists $\delta^{\sim}>\sim \overline{0}$ such that $\overline{0}<\sim \tilde{x}-a^{\sim}<\sim \tilde{\delta}$ implies

$$
\phi(\tilde{x})-\phi\left(a^{\sim}\right)<\tilde{\gamma}
$$

Moreover, $\phi: \mathbb{R}^{+}(\mathscr{P}) \rightarrow \mathbb{R}^{+}(\mathscr{P})$ is called soft continuous at every point $\tilde{a}$ of $\mathbb{R}^{+}(\mathscr{P})$, then we call $\phi$ as a continuous mapping.

A soft set $(T, \mathscr{P})$ on $\Omega$ is called a soft point $[4,14]$, denoted by $\widetilde{x_{\tau}}$, if there is a unique $\tau \in \mathscr{P}$ such that $T(\tau)=$ $\{x\}$ for some $\tau \in \mathscr{P}$ and $T(\omega)=\phi$ for all $\omega \in \mathscr{P} \backslash\{\tau\}$.

Definition 2 (see). Let $\tilde{X}=(\mathrm{T}, \mathscr{P})$ be an absolute soft set, and let $\mathcal{S} \mathscr{P}(\tilde{X})$ be the collection of all soft points of $\tilde{X}$. A mapping $\tilde{d}: \mathcal{S} \mathscr{P}(\tilde{X}) \times \mathcal{S} \mathscr{P}(\tilde{X}) \rightarrow \mathbb{R}^{+}(\mathscr{P})$ is called a soft metric on $\tilde{\mathrm{X}}$ if $\tilde{d}$ satisfies the following conditions for all $\widetilde{x_{\tau_{1}}}, \widetilde{x_{\tau_{2}}}, \widetilde{x_{\tau_{3}}} \in \tilde{X}$ :

$$
(M 1) \tilde{d}\left(\widetilde{x_{\tau_{1}}}, x_{\tau_{2}} \sim\right) \geq \overline{0},
$$

$$
\begin{aligned}
& (M 2) \tilde{d}\left(\widetilde{x_{\tau_{1}}}, \widetilde{x_{\tau_{2}}}\right)=\overline{0} \text { if and only if } \widetilde{x_{\tau_{1}}}=\widetilde{x_{\tau_{2}}}, \\
& (M 3) \tilde{d}\left(\widetilde{x_{\tau_{1}}}, \widetilde{x_{\tau_{2}}}\right)=\tilde{d}\left(\widetilde{x_{\tau_{2}}}, \widetilde{x_{\tau_{1}}}\right), \\
& (M 4) \tilde{d}\left(\widetilde{x_{\tau_{1}}}, x_{\tau_{3}}^{\sim}\right) \leq \sim \tilde{d}\left(\widetilde{x_{\tau_{1}}}, \widetilde{x_{\tau_{2}}}\right)+\tilde{d}\left(\widetilde{x_{\tau_{2}}}, \widetilde{x_{\tau_{3}}}\right) .
\end{aligned}
$$

The triple $(\tilde{X}, \tilde{d}, \mathscr{P})$ is called a soft metric space, in short, s.m.s.

For the sake of simplicity, we set $\mathscr{M}:=(\tilde{X}, \tilde{d}, \mathscr{P})$.

Suppose $\mathscr{M}$ is a s.m.s. and $\tilde{\gamma}$ is a nonnegative soft real number. A soft open ball with the center $\tilde{x}_{e}$ and radius $\tilde{\gamma}$ is defined by $B\left(\tilde{x_{e}}, \tilde{\gamma}\right)=\left\{y_{e^{\prime}} \sim \epsilon^{\sim} \tilde{X}: \tilde{d}\left(\tilde{x_{e}}, y_{e^{\prime}} \sim\right)<\tilde{\gamma}\right\}$. Analogously, a soft closed ball with center $\widetilde{x_{e}}$ and radius $\tilde{\gamma}$ is $B\left[\widetilde{x_{e}}\right.$ $, \tilde{\gamma}]=\left\{y_{e^{\prime}} \sim \epsilon^{\sim} \tilde{X}: \tilde{d}\left(\tilde{x}_{e}, y_{e^{\prime}} \sim \leq^{\sim} \tilde{\gamma}\right\}\right.$. We set that a soft $\operatorname{set}(F, \mathscr{P})$ is soft open in $\tilde{X}$ with respect to $\tilde{d}$ if and only if all soft points of $(F, E)$ are interior points of $(F, \mathscr{P})$.

In a soft metric space $\mathscr{M}$, a sequence of soft points $\left\{\widetilde{x_{\lambda, n}}\right\}_{n}$ is called convergent in $\mathscr{M}$ if there is a soft point $y_{v}{ }^{\sim} \epsilon^{\sim} \tilde{X}$ such that

$$
\lim _{n \rightarrow \infty} \tilde{d}\left(\widetilde{x_{\lambda, n}}, \widetilde{y_{v}}\right)=\overline{0}
$$
$\mathscr{M}$ if

Furthermore, a sequence $\left\{\widetilde{x_{\lambda, n}}\right\}_{n}$ is said to be a Cauchy in

$$
\lim _{i, j \rightarrow \infty} \tilde{d}\left(\widetilde{x_{\lambda, i}}, \widetilde{x_{\lambda, j}}\right)=\overline{0}
$$

Moreover, if each Cauchy sequence in $\tilde{X}$ converges to some point of $\tilde{X}$, then $\mathscr{M}$ is called complete soft metric space.

Let $\mathcal{N}=\left(\tilde{Y}, \tilde{\sigma}, \mathscr{P}^{\prime}\right)$ be another soft metric space. A soft mapping $(f, \varphi): \mathscr{M} \rightarrow \mathscr{N}$ is soft continuous at a point $x_{\lambda} \epsilon^{\sim} \mathcal{S} \mathscr{P}(\tilde{X})$, if for each $B\left((f, \varphi)\left(\tilde{x}_{\lambda}\right), \tilde{\gamma}\right)$ of $\mathcal{N}$, there exists $\left.B\left(\widetilde{x_{\lambda}}\right), \tilde{\delta}\right)$ such that

$$
\left.f\left(B\left(\widetilde{x_{\lambda}}\right), \tilde{\delta}\right)\right) c^{\sim} B\left((f, \varphi)\left(\widetilde{x_{\lambda}}\right), \tilde{\gamma}\right) .
$$

In other words, for every $\gamma^{\sim}>\sim \overline{0}$, there exists $\delta^{\sim}>\sim \overline{0}$ such that $\tilde{d}\left(\tilde{x_{\lambda}}, y_{\mu} \sim\right)<\tilde{\delta}$ implies that $\tilde{\sigma}\left((f, \varphi)\left(\tilde{x_{\lambda}}\right),(f, \varphi)(\right.$ $\left.\left.x_{\lambda}{ }^{\sim}\right)\right)<\tilde{\gamma}$. Moreover, if $(f, \varphi)$ is soft continuous for each point of $\mathcal{S} \mathscr{P}(\tilde{X})$, then it is called soft continuous mapping.

\section{Soft Fixed Points for the Soft Inf- Comparable Contraction}

In this section, we first introduce the notion of soft inf -comparable mapping $\psi: \mathbb{R}^{+}(\mathscr{P}) \rightarrow \mathbb{R}^{+}(\mathscr{P})$.

Definition 3 (see [15]). Let $\mathscr{P}$ be a parameter set and $\psi: \mathbb{R}^{+}$ $(\mathscr{P}) \rightarrow \mathbb{R}^{+}(\mathscr{P})$. We call $\psi$ a soft inf-comparable mapping if it satisfies the following two axioms:

$\left(\psi_{1}\right) \psi\left(\tau^{\sim}\right)<^{\sim} \tilde{\tau}$ for all $\tilde{\tau} \in \mathbb{R}^{+}(\mathscr{P}) \backslash\{\overline{0}\}$ and $\psi(\overline{0})=\overline{0}$,

$\left(\psi_{2}\right) \lim \inf _{\widetilde{\tau_{n} \rightarrow \tilde{\tau}}} \psi\left(\tau_{n}^{\sim}\right)<\sim \tilde{\tau}$ for all $\tau^{\sim}>\sim \overline{0}$. 
Lemma 4. Let $\psi: \mathbb{R}^{+}(\mathscr{P}) \rightarrow \mathbb{R}^{+}(\mathscr{P})$ be a soft inf-comparable mapping. Then, $\lim _{n \rightarrow \infty} \psi^{n}(\tilde{\tau})=\overline{0}$ for all $\tilde{\tau}>\overline{0}$, where $\psi^{n}$ denotes the $n$-th iteration of $\psi$.

Proof. Let $\tau^{\sim}>\sim \overline{0}$ be fixed. If $\psi_{0}^{n}(\tilde{\tau})=\overline{0}$ for some $n_{0} \in \mathbb{N}$, then we have

$$
\psi^{n_{0}+1}(\tilde{\tau})=\psi\left(\psi^{n_{0}}(\tilde{\tau})\right)=\psi(\overline{0})=\overline{0}
$$

which implies that

$$
\psi^{n_{0}+r}(\tilde{\tau})=\overline{0}, \quad \text { for all } r \in \mathbb{N}
$$

Thus, we conclude that

$$
\lim _{n \rightarrow \infty} \psi^{n}(\tilde{\tau})=\overline{0}
$$
and

If $\psi^{n}\left(\tau^{\sim}\right)>\sim \overline{0}$ for each $n \in \mathbb{N}$, then we take $\widetilde{\sigma_{n}}=\psi^{n}(\tilde{\tau})$,

$$
\widetilde{\sigma_{n+1}}=\psi^{n+1}(\tilde{\tau})=\psi\left(\psi^{n}(\tilde{\tau})\right)=\psi\left(\widetilde{\sigma_{n}}\right)
$$

for all $n \in \mathbb{N}$. By the condition $\left(\psi_{1}\right)$ of the soft inf-comparable mapping $\psi$, we have that for all $n \in \mathbb{N}$,

$$
\widetilde{\sigma_{n+1}}=\psi\left(\sigma_{n}^{\sim}\right)<\widetilde{\sigma_{n}}
$$

Keeping $\left(\psi_{2}\right)$ in mind and considering that the soft sequence $\left\{\sigma_{n \in \mathbb{N}}\right\}$ is bounded from below and also that the soft sequence is strictly decreasing, one can find an $\nu^{\sim} \geq \overline{0}$ such that

$$
\lim _{n \rightarrow \infty} \widetilde{\sigma_{n}}=\tilde{v}
$$
find

We assert that $\tilde{v}=\overline{0}$. If not, suppose that $\nu^{\sim}>^{\sim} \overline{0}$, then we

$$
\tilde{v}=\lim _{n \rightarrow \infty} \widetilde{\sigma_{n+1}}=\lim _{n \rightarrow \infty} \inf \psi\left(\widetilde{\sigma_{n}}\right)=\lim _{\widetilde{\sigma}_{n} \rightarrow \tilde{v}} \inf \psi\left(\sigma_{n}{ }^{\sim}\right)<\tilde{v}
$$

a contradiction. So we obtain that $\lim _{n \rightarrow \infty} \psi^{n}(\tilde{\tau})=\overline{0}$.

We introduce the notion of soft inf-comparable contraction, as follows:

Definition 5. Let $\mathscr{M}$ be a soft metric space and let $\psi: \mathbb{R}^{+}$ $(\mathscr{P}) \rightarrow \mathbb{R}^{+}(\mathscr{P})$ be a soft inf-comparable mapping. A mapping $(f, \varphi): \mathscr{M} \rightarrow \mathscr{M}$ is called a soft inf-comparable contraction if for each soft points $\tilde{x}_{p}, \tilde{y_{\tau}} \in \mathcal{S} \mathscr{P}(\tilde{X})$,

$$
\begin{aligned}
& \tilde{d}\left((f, \varphi)\left(\tilde{x}_{p}\right),(f, \varphi)\left(y_{\tau}^{\sim}\right)\right) \\
& \leq^{\sim} \psi\left(\max \left\{\tilde{d}\left(\tilde{x_{p}}, \tilde{y_{\tau}}\right), \tilde{d}\left(\tilde{x_{p}},(f, \varphi)\left(\widetilde{x_{p}}\right)\right), \tilde{d}\left(\tilde{y_{\tau}},(f, \varphi)\left(\tilde{y_{\tau}}\right)\right)\right\}\right) .
\end{aligned}
$$

Example 6. Set $\mathscr{R}=(\widetilde{\mathbb{R}}, \tilde{d}, \mathscr{P})$ where the soft metric is expressed as

$$
\begin{aligned}
d_{\varphi}(p, \tau) & =\max \{p, \tau\}, d(x, y)=|x-y|, \\
\tilde{d}\left(\tilde{x}_{p}, \tilde{y}_{\tau}\right) & =\frac{3}{5} d_{\varphi}(p, \tau)+d(x, y),
\end{aligned}
$$

with $\mathscr{P}=[0, \infty), \varphi(t)=(2 / 3) t$ for $t \in[0, \infty)$.

Let $\psi: \mathbb{R}^{+}(\mathscr{P}) \rightarrow \mathbb{R}^{+}(\mathscr{P})$ be denoted by

$$
\psi(\tilde{\omega})=\frac{5}{6} \tilde{\omega}
$$

and let $f(x)=(2 / 5) x$. Consequently, we find

$$
\begin{aligned}
& \tilde{d}\left((f, \varphi)\left(\tilde{x}_{p}\right),(f, \varphi)\left(\widetilde{y}_{\tau}\right)\right)=\tilde{d}\left(\frac{2}{5} \widetilde{\left.x_{(2 / 3)}\right)}, \frac{2}{5} y_{(2 / 3) \tau}\right)=\frac{2}{5} \max \{p, \tau\}+\frac{2}{5}|x-y|, \\
& \tilde{d}\left(\widetilde{x}_{p}, \tilde{y}_{\tau}\right)=\frac{3}{5} \max \{p, \tau\}+|x-y|, \\
& \tilde{d}\left(\widetilde{x_{p}}, \frac{1}{2} \widetilde{x_{(1 / 3)}}\right)=\frac{3}{5} \max \left\{p, \frac{1}{3} p\right\}+\left|x-\frac{1}{2} x\right|=\frac{3}{5} p+\frac{1}{2}|x| \text {, } \\
& \tilde{d}\left(\widetilde{y_{\tau}}, \frac{1}{2} y_{(1 / 3) \tau}\right)=\frac{3}{5} \max \left\{\tau, \frac{1}{3} \tau\right\}+\left|y-\frac{1}{2} y\right|=\frac{3}{5} \tau+\frac{1}{2}|y| .
\end{aligned}
$$

As a result, $(f, \varphi)$ forms a soft inf-comparable contraction on $\mathscr{R}$.

We say that a soft point $\tilde{x_{\tau}} \in \mathcal{S} \mathscr{P}(\tilde{X})$ is a soft fixed point of a self-soft-mapping $(f, \varphi)$ if $(f, \varphi)\left(\tilde{x_{\tau}}\right)=\tilde{x_{\tau}}$.

Theorem 7. Let $\psi: \mathbb{R}^{+}(\mathscr{P}) \rightarrow \mathbb{R}^{+}(\mathscr{P})$ be a soft inf-comparable mapping. Let $(f, \varphi): \mathscr{M} \rightarrow \mathscr{M}$ be a soft inf-comparable contraction on a complete soft metric space $\mathscr{M}$. Then, a soft mapping $(f, \varphi)$ possesses a soft fixed point.

Proof. Let $\widetilde{x_{\tau_{0}}^{0}} \in \mathcal{S} \mathscr{P}(\tilde{X})$ be given. For each $n \in \mathbb{N} \cup\{0\}$, we put

$$
\widetilde{x_{\tau_{n+1}}^{n+1}}=\left((f, \varphi)\left(\widetilde{x_{\tau_{n}}^{n}}\right)\right)=\left(f^{n+1}\left(\widetilde{x_{\tau_{0}}^{0}}\right)\right)_{\varphi^{n+1}\left(\tau_{0}\right)} .
$$

Then, we have for each $n \in \mathbb{N} \cup\{0\}$

$$
\begin{aligned}
\tilde{d}\left(\widetilde{x_{\tau_{n}}^{n}}, \widetilde{x_{\tau_{n+1}}^{n+1}}\right)= & \tilde{d}\left((f, \varphi)\left(\widetilde{x_{\tau_{n-1}^{n-1}}^{n-1}}\right),(f, \varphi)\left(x_{\tau_{n}}^{n}\right)\right) \\
\leq & \psi\left(\operatorname { m a x } \left\{\tilde{d}\left(\widetilde{x_{\tau_{n-1}^{n-1}}^{n-}}, \widetilde{x_{\tau_{n}}^{n}}\right), \tilde{d}\left(\widetilde{x_{\tau_{n-1}^{n-1}}^{n-1}},(f, \varphi)\left(\widetilde{x_{\tau_{n-1}^{n-1}}^{n-1}}\right)\right), \tilde{d}\right.\right. \\
& \left.\left.\cdot\left(\widetilde{x_{\tau_{n}}^{n}},(f, \varphi)\left(\widetilde{x_{\tau_{n}}^{n}}\right)\right)\right\}\right)=\psi\left(\operatorname { m a x } \left\{\tilde{d}\left(\widetilde{x_{\tau_{n-1}^{n-1}}^{n-1}}, \widetilde{x_{\tau_{n}}^{n}}\right), \tilde{d}\right.\right. \\
& \left.\left.\cdot\left(\widetilde{x_{\tau_{n-1}^{n-1}}^{n-1}} \widetilde{x_{\tau_{n}}^{n}}\right), \tilde{d}\left(\widetilde{x_{\tau_{n}}^{n}}, \widetilde{x_{\tau_{n+1}}^{n+1}}\right)\right\}\right) .
\end{aligned}
$$

Since $\psi: \mathbb{R}^{+}(\mathscr{P}) \rightarrow \mathbb{R}^{+}(\mathscr{P})$ is a soft inf-comparable mapping, we can conclude that for each $n \in \mathbb{N} \cup\{0\}$,

$$
\tilde{d}\left(\widetilde{x_{\tau_{n}}^{n}}, x_{\tau_{n+1}^{n+1}}^{n+}\right) \leq \sim\left(\tilde{d}\left(\widetilde{x_{\tau_{n-1}^{n-1}}^{n-1}}, \widetilde{x_{\tau_{n}}^{n}}\right)\right) .
$$


By induction, we obtain that

$$
\begin{aligned}
\tilde{d}\left(\widetilde{x_{\tau_{n}}^{n}}, x_{\tau_{n+1}}^{n+1}\right) & \leq \sim \psi\left(\tilde{d}\left(\widetilde{x_{\tau_{n-1}^{n-1}}^{n-1}}, x_{\tau_{n}}^{n \sim}\right)\right) \leq \sim \psi^{2}\left(\tilde{d}\left(\widetilde{x_{\tau_{n-2}}^{n-2}}, x_{\tau_{n-1}^{n-1}}^{n-}\right)\right) \\
& \leq \sim \cdots \operatorname{s}^{n}\left(\tilde{d}\left(\widetilde{x_{\tau_{0}}^{0}}, \widetilde{x_{\tau_{1}}^{1}}\right)\right) .
\end{aligned}
$$

By Lemma 4, we obtained that

$$
\lim _{n \rightarrow \infty} \tilde{d}\left(\widetilde{x_{\tau_{n}}^{n}}, \widetilde{x_{\tau_{n+1}+1}^{n+1}}\right)=\overline{0}
$$

In what follows, we check whether the sequence $\left\{\widetilde{x_{\tau_{n}}^{n}}\right\}$ is Cauchy: for each $\tilde{\varepsilon}$, there is $n_{0} \in \mathbb{N}$ such that if $n, k \geq n_{0}$, then

$$
\tilde{d}\left(\widetilde{x_{\tau_{k_{r}}}^{k_{r}}}, x_{\tau_{n_{r}}}^{n_{r} \sim}\right)<\tilde{\mathcal{\varepsilon}} .
$$

Suppose, on the contrary, that the statement $(*)$ is false. Then, there exists $\mathcal{E}^{\sim}>\overline{0}$ such that, for any $r \in \mathbb{N}$, there are $n_{r}, k_{r} \in \mathbb{N}$ with $n_{r}>k_{r} \geq r$ satisfying that

(1) $n_{r}$ is even and $k_{r}$ is odd

(2) $\tilde{d}\left(\widetilde{x_{\tau_{k_{r}}}^{k_{r}}}, x_{\tau_{n_{r}}}^{n_{r} \sim}\right) \geq \sim \tilde{\mathcal{E}}$

(3) $n_{r}$ is the smallest even number such that condition (2) holds

By (1) and (2), we conclude that

$$
\begin{aligned}
& \mathcal{\varepsilon}^{\sim} \leq \tilde{d}\left(\widetilde{x_{\tau_{k_{r}}}^{k_{r}}}, x_{\tau_{n_{r}}}^{n_{r_{r}} \sim}\right) \leq \sim \tilde{d}\left(\widetilde{x_{\tau_{k_{r}}}^{k_{r}}}, \widetilde{x_{\tau_{n_{r}-2}}^{n_{r^{\prime}}-2}}\right)+\tilde{d}\left(\widetilde{x_{\tau_{n_{r}-2}}^{n_{r}-2}}, \widetilde{x_{\tau_{n_{r}-1}}^{n_{r}-1}}\right)
\end{aligned}
$$

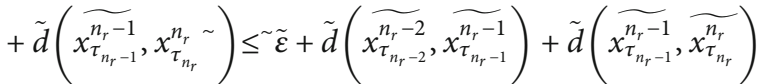

Letting $r \rightarrow \infty$, we obtain that

$$
\lim _{r \rightarrow \infty} \tilde{d}\left(\widetilde{x_{\tau_{k_{r}}}^{k_{r}}}, \widetilde{x_{\tau_{n_{r}}}^{n_{r}}}\right)=\tilde{\varepsilon} .
$$

On the other hand,

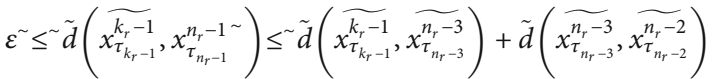

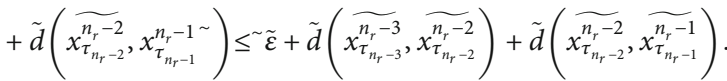

Letting $r \rightarrow \infty$, we obtain that

$$
\lim _{r \rightarrow \infty} \tilde{d}\left(\widetilde{x_{\tau_{k_{r}-1}}^{k_{r}-1}}, \widetilde{x_{\tau_{n_{r}-1}}^{n_{r}-1}}\right)=\tilde{\varepsilon} .
$$

By the above arguments, we obtain that

$$
\begin{aligned}
& \tilde{d}\left(\widetilde{x_{\tau_{k_{r}}}^{k_{r}}}, \widetilde{x_{\tau_{n_{r}}}^{n_{r}}}\right)=\tilde{d}\left((f, \varphi)(f, \varphi),(f, \varphi)\left(x_{\tau_{n_{r}-1}^{n_{r}-1}}^{n}\right)\right) \\
& \leq^{\sim} \psi\left(\operatorname { m a x } \left\{\tilde{d}\left(\widetilde{x_{\tau_{k_{r}-1}}^{k_{r}-1}}, \widetilde{x_{\tau_{n_{r}-1}}^{n_{n_{r}}-1}}\right), d\right.\right. \\
& \cdot\left(\widetilde{x_{\tau_{k_{r}-1}}^{k_{k-1}}},(f, \varphi)\left(\widetilde{x_{\tau_{k r-1}}^{k_{r}-1}}\right)\right), d \\
& \left.\left.\cdot\left(\widetilde{x_{\tau_{n_{r}-1}}^{n_{n^{\prime}-1}}},(f, \varphi)\left(x_{\tau_{n_{r}-1}^{n_{r}-1}}\right)\right)\right\}\right) \\
& \leq^{\sim} \psi\left(\operatorname { m a x } \left\{\tilde{d}\left(\widetilde{x_{\tau_{k_{r}-1}}^{k_{r}-1}}, \widetilde{x_{\tau_{n_{r}-1}}^{n_{n-1}}}\right), \tilde{d}\left(\widetilde{x_{\tau_{k_{r}-1}-1}^{k_{k_{r}}}}, \widetilde{x_{\tau_{r r}}}\right), d\right.\right. \\
& \left.\left.\cdot\left(\widetilde{x_{n_{n_{r}-1}}^{n_{r}-1}}, \widetilde{x_{\tau_{n_{r}}}^{n_{r}}}\right)\right\}\right)
\end{aligned}
$$

Taking $\lim _{r \rightarrow \infty}$ inf, we get $\mathcal{\varepsilon}^{\sim}<^{\sim} \tilde{\mathcal{E}}$. This implies a contradiction. So the sequence $\left\{\widetilde{x_{\tau_{n}}^{n}}\right\}$ is Cauchy.

Since $\mathscr{M}$ is complete, there exists $\widetilde{x_{\tau}^{*}} \in \tilde{X}$ such that

$$
\widetilde{x_{\tau_{n}}^{n}} \rightarrow \widetilde{x_{\tau}^{*}} \text { as } n \rightarrow \infty
$$

that is,

$$
\tilde{d}\left(\widetilde{x_{\tau_{n}}^{n}}, \widetilde{x_{\tau}^{*}}\right) \rightarrow \overline{0} \text { as } n \rightarrow \infty
$$

Notice also that

$$
\begin{aligned}
& \tilde{d}\left((f, \varphi)\left(\widetilde{x_{\tau}^{*}}\right), x_{\tau}^{* \sim}\right) \leq \tilde{d}\left((f, \varphi)\left(\widetilde{x_{\tau_{n}}^{n}}\right),(f, \varphi)\left(\widetilde{x_{\tau}^{*}}\right)\right) \\
&+\tilde{d}\left((f, \varphi)\left(\widetilde{x_{\tau_{n}}^{n}}\right), x_{\tau}^{* \sim}\right) \\
&<\sim\left(\operatorname { m a x } \left\{\tilde{d}\left(\widetilde{x_{\tau_{n}}^{n}}, \widetilde{x_{\tau}^{*}}\right), \tilde{d}\left(\widetilde{x_{\tau_{n}}^{n}},(f, \varphi)\left(\widetilde{x_{\tau_{n}}^{n}}\right)\right), \tilde{d}\left(\widetilde{x_{\tau}^{*}},(f, \varphi)\right.\right.\right. \\
&\left.\left.\left.\cdot\left(\widetilde{x_{\tau}^{*}}\right)\right)\right\}\right)+\tilde{d}\left(\widetilde{x_{\tau_{n+1}^{n+1}}^{n+1}}, x_{\tau}^{* \sim}\right) \\
&<\sim\left(\max \left\{\tilde{d}\left(\widetilde{x_{\tau_{n}}^{n}}, \widetilde{x_{\tau}^{*}}\right), \tilde{d}\left(\widetilde{x_{\tau_{n}}^{n}}, \widetilde{x_{\tau_{n+1}+1}^{n+1}}\right), \tilde{d}\left(\widetilde{x_{\tau}^{*}},(f, \varphi)\left(\widetilde{x_{\tau}^{*}}\right)\right)\right\}\right) \\
&+\tilde{d}\left(\widetilde{x_{\tau_{n+1}^{n+1}}^{n+1}}, \widetilde{x_{\tau}^{*}}\right) .
\end{aligned}
$$

Taking $n \rightarrow \infty$, we get that

$$
\begin{aligned}
& \tilde{d}\left((f, \varphi)\left(\widetilde{x_{\tau}^{*}}\right), x_{\tau}^{* \sim}\right) \\
& \quad \leq \sim \psi\left(\max \left\{\overline{0}, \overline{0}, \tilde{d}\left(\widetilde{x_{\tau}^{*}},(f, \varphi)\left(\widetilde{x_{\tau}^{*}}\right)\right)\right\}\right)+\overline{0}<\tilde{d}\left((f, \varphi)\left(\widetilde{x_{\tau}^{*}}\right), \widetilde{x_{\tau}^{*}}\right),
\end{aligned}
$$

and this is a contradiction unless $\tilde{d}\left((f, \varphi)\left(\tilde{x}_{\tau}^{*}\right), \tilde{x}_{\tau}^{*}\right)=\overline{0}$. Thus, $(f, \varphi)\left(\tilde{x}_{\tau}^{*}\right)=\tilde{x}_{\lambda}^{*}$ completes the proof.

Example 8. Consider Example 6. All hypotheses of Theorem 7 are fulfilled. Thus, we can conclude that $\overline{0_{0}}$ is a fixed soft 
point of the soft inf-comparable Meir-Keeler contraction $(f, \varphi)$.

\section{Observation on the Soft Comparable Meir- Keeler Contractions}

We start this section by recalling the Meir-Keeler contraction in the standard setting.

Definition 9. (see). A self-mapping $g$ on a metric space $(X, d)$ is called a Meir-Keeler contraction if the following is fulfilled: for any $\eta>0$, there is $\gamma>0$ such that

$$
\eta \leq d(x, y)<\eta+\gamma \Rightarrow d(g x, g y)<\eta \quad \text { for all } x, y \in X .
$$

The mapping $\phi: \mathbb{R}^{+}(\mathscr{P}) \times \mathbb{R}^{+}(\mathscr{P}) \times \mathbb{R}^{+}(\mathscr{P}) \rightarrow \mathbb{R}^{+}(\mathscr{P})$ is said to be soft comparable, if the following two axioms are fulfilled:

$\left(\phi_{1}\right) \phi$ is a soft increasing and soft continuous function in each coordinate

$\left(\phi_{2}\right)$ for $\tilde{\omega} \in \mathbb{R}^{+}(\mathscr{P}) \backslash\{\overline{0}\}, \phi\left(\tilde{\omega}, \tilde{\omega}, \omega^{\sim}\right)<\sim \tilde{\omega}$, and $\phi\left(\widetilde{\omega_{1}}, \widetilde{\omega_{2}}\right.$ ,$\left.\widetilde{\omega_{3}}\right)=\overline{0}$ if and only if $\widetilde{\omega_{1}}=\widetilde{\omega_{2}}=\widetilde{\omega_{3}}=\overline{0}$

Now, we introduce the notion of soft comparable MeirKeeler contraction.

Definition 10. Let $\phi: \mathbb{R}^{+}(\mathscr{P}) \times \mathbb{R}^{+}(\mathscr{P}) \times \mathbb{R}^{+}(\mathscr{P}) \rightarrow \mathbb{R}^{+}(\mathscr{P})$ be soft comparable. A self-soft-mapping $(f, \varphi)$ on a soft metric space $\mathscr{M}$ is called a soft comparable Meir-Keeler contraction if for each soft real number $\eta^{\sim}>\overline{0}$, there is $\gamma^{\sim}>\sim \overline{0}$ such that

$$
\begin{aligned}
\eta^{\sim} & \leq \sim \phi\left(\tilde{d}\left(\widetilde{x_{p}}, \widetilde{y_{\tau}}\right), \tilde{d}\left(\widetilde{x_{p}},(f, \varphi)\left(\widetilde{x_{p}}\right)\right), \tilde{d}\left(\widetilde{y}_{\tau},(f, \varphi)\left(y_{\tau} \sim\right)\right)\right) \\
& <\tilde{\eta}+\tilde{\gamma} \Rightarrow \tilde{d}\left((f, \varphi)\left(\widetilde{x_{p}}\right),(f, \varphi)\left(y_{\tau}\right)\right)<\tilde{\eta},
\end{aligned}
$$

for each soft points $\widetilde{x_{\lambda}}, \widetilde{y_{\mu}} \in \mathcal{S} \mathscr{P}(\tilde{X})$.

Example 11. Set $\mathscr{R}=(\widetilde{\mathbb{R}}, \tilde{d}, \mathscr{P})$ where the soft metric is expressed as

$$
\begin{aligned}
d_{\varphi}(p, \tau) & =\max \{p, \tau\}, d(x, y)=|x-y|, \\
\tilde{d}\left(\widetilde{x}_{p}, \widetilde{y}_{\tau}\right) & =d_{\varphi}(p, \tau)+d(x, y),
\end{aligned}
$$

with $\mathscr{P}=[0, \infty), \varphi(t)=(1 / 3) t$ for $t \in[0, \infty)$. by

Let $\phi: \mathbb{R}^{+}(\mathscr{P}) \times \mathbb{R}^{+}(\mathscr{P}) \times \mathbb{R}^{+}(\mathscr{P}) \rightarrow \mathbb{R}^{+}(\mathscr{P})$ be denoted

$$
\phi\left(\widetilde{\omega_{1}}, \widetilde{\omega_{2}}, \widetilde{\omega_{3}}\right)=\frac{3}{4} \cdot \max \left\{\widetilde{\omega_{1}}, \widetilde{\omega_{2}}, \widetilde{\omega_{3}}\right\},
$$

where

$$
\begin{aligned}
& \widetilde{\omega_{1}}=\tilde{d}\left(\widetilde{x}_{p}, \widetilde{y}_{\tau}\right), \\
& \widetilde{\omega_{2}}=\tilde{d}\left(\widetilde{x_{p}},(f, \varphi)\left(\widetilde{x_{p}}\right)\right), \\
& \widetilde{\omega_{3}}=\tilde{d}\left(\widetilde{y_{\tau}},(f, \varphi)\left(\widetilde{y_{\tau}}\right)\right) .
\end{aligned}
$$

Let $f(x)=(1 / 2) x$. Then,

$$
\begin{aligned}
\tilde{d}\left((f, \varphi)\left(\widetilde{x}_{p}\right),(f, \varphi)\left(\widetilde{y_{\tau}}\right)\right) & =\tilde{d}\left(\frac{1}{2} x_{(1 / 3) p}, \frac{1}{2} y_{(1 / 3) \tau}\right) \\
& =\frac{1}{3} \max \{p, \tau\}+\frac{1}{2}|x-y|,
\end{aligned}
$$

$\tilde{\omega}_{1}=\tilde{d}\left(\widetilde{x_{p}}, \tilde{y}_{\tau}\right)=\max \{p, \tau\}+|x-y|$,

$\widetilde{\omega_{2}}=\tilde{d}\left(\widetilde{x_{p}}, \frac{1}{2} x_{(1 / 3) p}\right)=\max \left\{p, \frac{1}{3} p\right\}+\left|x-\frac{1}{2} x\right|=p+\frac{1}{2}|x|$,

$\widetilde{\omega_{3}}=\tilde{d}\left(\widetilde{y_{\tau}}, \frac{1}{2} \frac{y_{(1 / 3) \tau}}{2}\right)=\max \left\{\tau, \frac{1}{3} \tau\right\}+\left|y-\frac{1}{2} y\right|=\tau+\frac{1}{2}|y|$.

So we can conclude that

$$
\begin{aligned}
& \phi\left(\widetilde{\omega_{1}}, \widetilde{\omega_{2}}, \widetilde{\omega_{3}}, \widetilde{t_{4}}\right) \\
& \quad=\frac{3}{4} \cdot \max \left\{\widetilde{\omega_{1}}, \widetilde{\omega_{2}}, \omega_{3}^{\sim}\right\} \geq^{\sim} \frac{3}{4} \widetilde{\omega_{1}}=\frac{3}{4}(\max \{p, \tau\}+|x-y|) .
\end{aligned}
$$

Consequently, a soft mapping $(f, \varphi)$ forms a soft comparable Meir-Keeler contraction on $\mathscr{R}$.

We establish the following fixed point results for the soft comparable Meir-Keeler contraction.

Theorem 12. Let $\mathscr{M}$ be a complete soft metric space, and let $\phi: \mathbb{R}^{+}(\mathscr{P}) \times \mathbb{R}^{+}(\mathscr{P}) \times \mathbb{R}^{+}(\mathscr{P}) \rightarrow \mathbb{R}^{+}(\mathscr{P})$ be a soft comparable. Let $(f, \varphi): \mathscr{M} \rightarrow \mathscr{M}$ be a soft comparable Meir-Keeler contraction on $\mathscr{M}$. Then, $(f, \varphi)$ possesses a soft fixed point.

Proof. Let $\widetilde{x_{\tau_{0}}^{0}} \in \mathcal{S P}(\tilde{X})$ be given. For each $n \in \mathbb{N} \cup\{0\}$, we put

$$
\widetilde{x_{\tau_{n+1}}^{n+1}}=\left((f, \varphi)\left(\widetilde{x_{\tau_{n}}^{n}}\right)\right)=\left(f^{n+1}\left(\widetilde{x_{\tau_{0}}^{0}}\right)\right)_{\varphi^{n+1}\left(\tau_{0}\right)} .
$$

So, for each $n \in \mathbb{N} \cup\{0\}$ we have

$$
\begin{aligned}
& \tilde{d}\left(\widetilde{x_{\tau_{n}}^{n}}, \widetilde{x_{n+1}^{n+1}}\right)=\tilde{d}\left((f, \varphi)\left(\widetilde{x_{\tau_{n-1}}^{n-1}}\right),(f, \varphi)\left(x_{\tau_{n}}^{n}\right)\right) \\
& \quad \leq \sim \phi\left(\tilde{d}\left(\widetilde{x_{\tau_{n-1}}^{n-1}}, \widetilde{x_{\tau_{n}}^{n}}\right), \tilde{d}\left(\widetilde{x_{\tau_{n-1}}^{n-1}},(f, \varphi)\left(\widetilde{x_{\tau_{n-1}}^{n-1}}\right)\right), \tilde{d}\left(\widetilde{x_{\tau_{n}}^{n}},(f, \varphi)\left(\widetilde{x_{\tau_{n}}^{n}}\right)\right)\right) \\
& \quad=\phi\left(\tilde{d}\left(\widetilde{x_{\tau_{n-1}}^{n-1}}, \widetilde{x_{\tau_{n}}^{n}}\right), \tilde{d}\left(\widetilde{x_{\tau_{n-1}}^{n-1}}, \widetilde{x_{\tau_{n}}^{n}}\right), \tilde{d}\left(\widetilde{x_{\tau_{n}}^{n}}, \widetilde{x_{\tau_{n+1}}^{n+1}}\right)\right) .
\end{aligned}
$$


If $\tilde{d}\left(\widetilde{x_{\tau_{k-1}-1}^{k-1}}, x_{\tau_{k}}^{k \sim}\right)<\sim \tilde{d}\left(\widetilde{x_{\tau_{k}}^{k}}, \widetilde{x_{\tau_{k+1}}^{k+1}}\right)$ for some $k \in \mathbb{N}$, then by the above inequality and the conditions of the function $\phi$, we have

$$
\begin{aligned}
& \tilde{d}\left(\widetilde{x_{\tau_{n}}^{k}}, x_{\tau_{k+1}^{k+1}}^{\sim}\right) \leq \sim \phi\left(\tilde{d}\left(\widetilde{x_{\tau_{k-1}}^{k-1}}, \widetilde{x_{\tau_{k}}^{k}}\right), \tilde{d}\left(\widetilde{x_{\tau_{k-1}}^{k-1}}, \widetilde{x_{\tau_{k}}^{k}}\right), \tilde{d}\left(\tilde{k}_{\tau_{k}}^{n}, x_{\tau_{k+1}}^{k+1}\right)\right) \\
& \quad<\tilde{d}\left(\widetilde{x_{\tau_{k}}^{k}}, \widetilde{x_{\tau_{k+1}}^{k+1}}\right),
\end{aligned}
$$
find

which implies a contradiction. Hence, for each $n \in \mathbb{N}$, we

$$
\tilde{d}\left(\widetilde{x_{\tau_{n}}^{n}}, x_{\tau_{n+1}^{n+1}}^{n}\right)<\tilde{d}\left(\widetilde{x_{\tau_{k-1}^{k-1}}^{k-1}}, \widetilde{x_{\tau_{k}}^{k}}\right) .
$$

Thus, the sequence $\left\{\tilde{d}\left(\widetilde{x_{\tau_{n}}^{n}}, \widetilde{x_{\tau_{n+1}+1}^{n+1}}\right)\right\}$ is decreasing and converges to a soft real number, say $\gamma^{\sim} \geq \overline{0}$. In other words, $\tilde{d}\left(\widetilde{x_{\tau_{n}}^{n}}, \widetilde{x_{\tau_{n+1}+1}^{n+1}}\right) \rightarrow \tilde{\gamma}$, as $n \rightarrow \infty$.

Notice that $\tilde{\gamma}=\inf \left\{\tilde{d}\left(\widetilde{x_{\tau_{n}}^{n}}, \widetilde{x_{\tau_{n+1}}^{n+1}}\right): n \in \mathbb{N} \cup\{0\}\right\}$. We claim that $\tilde{\gamma}=\overline{0}$. Suppose, on the contrary, that $\gamma^{\sim}>\overline{0}$. Since $(f, \varphi)$ is a soft comparable Meir-Keeler contraction, corresponding to $\tilde{\gamma}$, there exists $\eta^{\sim}>\overline{0}$ and $k \in \mathbb{N}$ such that

$$
\begin{aligned}
\gamma^{\sim} & \leq^{\sim} \phi\left(\tilde{d}\left(\widetilde{x_{\tau_{k}}^{k}}, \widetilde{x_{\tau_{k+1}}^{k+1}}\right), \tilde{d}\left(\widetilde{x_{\tau_{k}}^{k}}, \widetilde{x_{\tau_{k+1}}^{k+1}}\right), \tilde{d}\left(\widetilde{x_{\tau_{k+1}}^{k+1}}, x_{\tau_{k+2}}^{k+2}\right)\right)<\sim \tilde{\gamma}+\tilde{\eta} \\
& \Rightarrow \tilde{d}\left(\widetilde{x_{\tau_{k+1}}^{k+1}}, \widetilde{x_{\tau_{k+2}}^{k+2}}\right)=\tilde{d}\left((f, \varphi)\left(\widetilde{x_{\tau_{k}}^{k}}\right),(f, \varphi)\left(x_{\tau_{k+1}^{k+1}}^{\sim}\right)\right)<\tilde{\gamma} .
\end{aligned}
$$

This is a contradiction since $\tilde{\gamma}=\inf \left\{\tilde{d}\left(\widetilde{x_{\tau_{n}}^{n}}, \widetilde{x_{\tau_{n+1}+1}^{n+1}}\right): n \in \mathbb{N}\right.$ $\cup\{0\}\}$. Thus, we obtain that $\tilde{d}\left(\widetilde{x_{\tau_{n}}^{n}}, \widetilde{x_{\tau_{n+1}}^{n+1}}\right) \rightarrow \overline{0}$, as $n \rightarrow \infty$.

As a next step, we check whether the sequence $\left\{\widetilde{x_{\tau_{n}}^{n}}\right\}$ is Cauchy in $\mathscr{M}$. Suppose, on the contrary, it is not. Thus, there exists a soft real number $\mathcal{E}^{\sim}>\sim \overline{0}$ such that for any $k \in \mathbb{N}$, there are $m_{k}, n_{k} \in \mathbb{N}$ with $n_{k}>m_{k} \geq k$ satisfying

$$
\tilde{d}\left(\widetilde{x_{\tau_{m_{k}}}^{m_{k}}}, x_{\tau_{n_{k}}}^{n_{n_{k}} \sim}\right) \geq \sim \tilde{\mathcal{E}}
$$

Further, corresponding to $m_{k} \geq k$, we can choose $n_{k}$ in such a way that it is the smallest integer with $n_{k}>m_{k} \geq k$ and $\tilde{d}\left(\widetilde{x_{\tau_{m_{k}}}^{m_{k}}}, x_{\tau_{n_{k}}}^{n_{k} \sim}\right) \geq \sim \widetilde{\mathcal{\varepsilon}}$. Therefore,

$$
\tilde{d}\left(\widetilde{x_{\tau_{m_{k}}}^{m_{k}}}, x_{\tau_{n_{k}-2}}^{n_{n_{k}-2}}\right)<\sim \widetilde{\varepsilon}
$$

So, we derive that

$$
\begin{aligned}
& \varepsilon^{\sim} \leq \tilde{d}\left(\widetilde{x_{\tau_{m_{k}}}^{m_{k}}}, x_{\tau_{n_{k}}}^{n_{k} \sim}\right) \leq \sim \tilde{d}\left(\widetilde{x_{\tau_{m_{k}}}^{m_{k}}}, \widetilde{x_{\tau_{n_{k}-2}}^{n_{k}-2}}\right)+\tilde{d}\left(\widetilde{x_{\tau_{n_{k}-2}}^{n_{k}-2}}, \widetilde{x_{\tau_{n_{k}-1}}^{n_{k}-1}}\right) \\
& +\tilde{d}\left(\widetilde{x_{\tau_{n_{k}-1}}^{n_{k_{k}-1}}, x_{\tau_{n_{k}}}^{n_{k}} \sim}\right)<\sim \tilde{\mathcal{\varepsilon}}+\tilde{d}\left(\widetilde{x_{\tau_{n_{k}-2}}^{n_{k}-2}}, \widetilde{x_{\tau_{n_{k}-1}}^{n_{k}-1}}\right)+\tilde{d}\left(\widetilde{x_{\tau_{n_{k}-1}}^{n_{k}-1}}, \widetilde{x_{\tau_{n_{k}}}^{n_{k}}}\right),
\end{aligned}
$$

for all $k \in \mathbb{N}$. As $k \rightarrow \infty$, the inequality above yields that

$$
\lim _{k \rightarrow \infty} \tilde{d}\left(\widetilde{x_{\tau_{m_{k}}}^{m_{k}}}, \widetilde{x_{\tau_{n_{k}}}^{n_{k}}}\right)=\tilde{\varepsilon}
$$

On the other hand, we have

$$
\begin{aligned}
& \mathcal{\varepsilon}^{\sim} \leq \tilde{d}\left(\widetilde{x_{\tau_{m_{k}}}^{m_{k}}}, x_{\tau_{n_{k}}}^{n_{k} \sim}\right) \leq \tilde{d}\left(\widetilde{x_{\tau_{m_{k}}}^{m_{k}}}, \widetilde{x_{\tau_{m_{k}+1}}^{m_{k}+1}}\right)+\tilde{d}\left(\widetilde{x_{\tau_{m_{k}+1}}^{m_{k}+1}}, \widetilde{x_{\tau_{n_{k}+1}}^{n_{k}+1}}\right) \\
& +\tilde{d}\left(\widetilde{x_{\tau_{n_{k}+1}}^{n_{k}+1}}, x_{\tau_{n_{k}}}^{n_{k}} \sim\right) \leq \tilde{d}\left(\widetilde{x_{\tau_{m_{k}}}^{m_{k}}}, \widetilde{x_{\tau_{m_{k}+1}}^{m_{k}+1}}\right)+\tilde{d}\left(\widetilde{x_{\tau_{m_{k}+1}}^{m_{k}+1}}, \widetilde{x_{\tau_{m_{k}}}^{m_{k}}}\right) \\
& +\tilde{d}\left(\widetilde{x_{\tau_{m_{k}}}^{m_{k}}}, \widetilde{x_{\tau_{n_{k}}}^{n_{k}}}\right)+\tilde{d}\left(\widetilde{x_{\tau_{n_{k}}}^{n_{k}}}, \widetilde{x_{\tau_{n_{k}+1}}^{n_{k}+1}}\right)+\tilde{d}\left(\widetilde{x_{\tau_{n_{k}+1}}^{n_{k}+1}}, \widetilde{x_{\tau_{n_{k}}}^{n_{k}}}\right) \text {. }
\end{aligned}
$$

Letting $k \rightarrow \infty$ in the above inequality, we get

$$
\lim _{k \rightarrow \infty} \tilde{d}\left(\widetilde{x_{\lambda_{m_{k}+1}}^{m_{k}+1}}, \widetilde{x_{\lambda_{n_{k}+1}+1}^{n_{k}+1}}\right)=\tilde{\varepsilon}
$$

Since $(f, \varphi)$ is a soft comparable Meir-Keeler contraction, we have

$$
\begin{aligned}
& \tilde{d}\left(\widetilde{x_{\tau_{m_{k}+1}}^{m_{k}+1}}, \widetilde{x_{\tau_{n_{k}+1}}^{n_{k}+1}}\right)=\tilde{d}\left((f, \varphi)\left(\widetilde{x_{\tau_{m_{k}}}^{m_{k}}}\right),(f, \varphi)\left(x_{\tau_{n_{k}}^{n_{k}}}^{n_{n}}\right)\right) \\
& \quad<\sim \phi\left(\tilde{d}\left(\widetilde{x_{\tau_{m_{k}}}^{m_{k}}}, \widetilde{x_{\tau_{n_{k}}}^{n_{k}}}\right), \tilde{d}\left(\widetilde{x_{\tau_{m_{k}}}^{m_{k}}}, \widetilde{x_{\tau_{m_{k}+1}}^{m_{m_{k}+1}}}\right), \tilde{d}\left(\widetilde{x_{\tau_{n_{k}}}^{n_{k}}}, \widetilde{x_{\tau_{n_{k}+1}}^{n_{n_{k}+1}}}\right)\right) .
\end{aligned}
$$

Moreover, since

$$
\begin{aligned}
& \tilde{d}\left(\widetilde{x_{\tau_{m_{k}}}^{m_{k}}}, x_{\tau_{n_{n_{k}+1}}^{n_{k}+1}}\right) \leq \tilde{d}\left(\tilde{x}_{\tau_{m_{k}}}^{m_{k}}, \widetilde{x_{\tau_{m_{k}+1}}^{m_{k}+1}}\right)+\tilde{d}\left(\widetilde{x_{\tau_{m_{k}+1}}^{m_{k}+1}}, \widetilde{x_{\tau_{n_{k}+1}}^{n_{k}+1}}\right), \tilde{d}\left(\widetilde{x_{\tau_{n_{k}}}^{n_{k}}}, x_{\tau_{m_{m_{k}+1}}^{m_{k}+1}}\right) \\
& \quad \leq \tilde{d}\left(\widetilde{x_{\tau_{n_{k}}}^{n_{k}}}, \widetilde{x_{\tau_{n_{k}+1}}^{n_{k}+1}}\right)+\tilde{d}\left(\widetilde{x_{\tau_{n_{k}+1}+1}^{n_{k}+1}}, \widetilde{\tau_{\tau_{m_{k}+1}}^{m_{k}+1}}\right) .
\end{aligned}
$$

Taking $k \rightarrow \infty$ in the above inequalities, we get that

$$
\mathcal{\varepsilon}^{\sim} \leq^{\sim} \phi\left(\tilde{\mathcal{\varepsilon}}, \tilde{\mathcal{\varepsilon}}, \mathcal{\varepsilon}^{\sim}\right)<\tilde{\mathcal{E}}
$$

and this is a contradiction. Thus, the sequence $\left\{\widetilde{x_{\lambda_{n}^{n}}}\right\}$ is Cauchy.

Keeping the completeness of $\mathscr{M}$ in mind, one can find $\widetilde{x_{\tau}^{*}} \in \tilde{X}$ such that

$$
\widetilde{x_{\tau_{n}}^{n}} \rightarrow \widetilde{x_{\tau}^{*}} \text { as } n \rightarrow \infty,
$$

that is,

$$
\tilde{d}\left(\widetilde{x_{\tau_{n}}^{n}}, \widetilde{x_{\tau}^{*}}\right) \rightarrow \overline{0} \text { as } n \rightarrow \infty \text {. }
$$


And, we also have

$$
\begin{aligned}
& \tilde{d}\left((f, \varphi)\left(\widetilde{x_{\tau}^{*}}\right), x_{\tau}^{* \sim}\right) \leq \sim \tilde{d}\left((f, \varphi)\left(\widetilde{x_{\tau_{n}}^{n}}\right),(f, \varphi)\left(\widetilde{x_{\tau}^{*}}\right)\right) \\
& \quad+\tilde{d}\left((f, \varphi)\left(\widetilde{x_{\tau_{n}}^{n}}\right), x_{\tau}^{* \sim}\right)<^{\sim} \phi\left(\tilde{d}\left(\widetilde{x_{\tau_{n}}^{n}}, \widetilde{x_{\tau}^{*}}\right), d\right. \\
& \left.\quad \cdot\left(\widetilde{x_{\tau_{n}}^{n}},(f, \varphi)\left(\widetilde{x_{\tau_{n}}^{n}}\right)\right), \tilde{d}\left(\widetilde{x_{\tau}^{*}},(f, \varphi)\left(\widetilde{x_{\tau}^{*}}\right)\right)\right) \\
& \quad+\tilde{d}\left(\widetilde{x_{\tau_{n+1}^{n+1}}}, x_{\tau}^{* \sim}\right)<_{\tau}^{\sim} \phi\left(\tilde{d}\left(\widetilde{x_{\tau_{n}}^{n}}, \widetilde{x_{\tau}^{*}}\right), \tilde{d}\left(\widetilde{x_{\tau_{n}}^{n}}, \widetilde{x_{\tau_{n+1}^{n+1}}^{n+1}}\right), d\right. \\
& \left.\quad \cdot\left(\widetilde{x_{\tau}^{*}},(f, \varphi)\left(\widetilde{x_{\tau}^{*}}\right)\right)\right)+\tilde{d}\left(\widetilde{x_{\tau_{n+1}^{n+1}}^{n+1}}, \widetilde{x_{\tau}^{*}}\right) .
\end{aligned}
$$

Taking $k \rightarrow \infty$ in the inequality above,

$$
\begin{gathered}
\tilde{d}\left((f, \varphi)\left(\widetilde{x_{\tau}^{*}}\right), x_{\tau}^{* \sim}\right) \leq \sim \phi\left(\overline{0}, \overline{0}, \tilde{d}(f, \varphi)\left(\widetilde{x_{\tau}^{*}}\right)\right)+\overline{0} \\
\leq^{\sim} \phi\left(\tilde{d}\left((f, \varphi)\left(\widetilde{x_{\tau}^{*}}\right), \widetilde{x_{\tau}^{*}}\right), \tilde{d}\left((f, \varphi)\left(\widetilde{x_{\tau}^{*}}\right), \widetilde{x_{\tau}^{*}}\right), d\right. \\
\left.\cdot\left((f, \varphi)\left(\widetilde{x_{\tau}^{*}}\right), x_{\tau}^{* \sim}\right)\right)<\tilde{d}\left((f, \varphi)\left(\widetilde{x_{\tau}^{*}}\right), \widetilde{x_{\tau}^{*}}\right),
\end{gathered}
$$

a contradiction unless $\tilde{d}\left((f, \varphi)\left(\widetilde{x_{\tau}^{*}}\right), \widetilde{x_{\tau}^{*}}\right)=\overline{0}$. Thus, $(f, \varphi)$ $\left(\tilde{x}_{\tau}^{*}\right)=\tilde{x}_{\tau}^{*}$ which completes the proof.

Example 13. Consider Example 11. One can easily check all hypotheses of Theorem 12 . Consequently, we conclude that $0_{0}$ is a fixed soft point of the soft comparable Meir-Keeler contraction $(f, \varphi)$.

We next introduce the notion of soft generalized MeirKeeler contraction, as follows:

Definition 14. Let $(\tilde{X}, \tilde{d}, \mathscr{P})$ be a soft metric space. A mapping $(f, \varphi):(\tilde{X}, \tilde{d}, \mathscr{P}) \rightarrow(\tilde{X}, \tilde{d}, \mathscr{P})$ is called a soft generalized Meir-Keeler contraction if for any soft real number $\eta^{\sim}>\overline{0}$, there exists $\gamma^{\sim}>\sim \overline{0}$ such that for each soft point $\tilde{x_{p}}, \widetilde{y_{\tau}} \in \mathcal{S} \mathscr{P}$ $(\tilde{X})$,

$$
\begin{aligned}
\eta^{\sim} & \leq \sim \max \left\{\tilde{d}\left(\tilde{x}_{p},(f, \varphi)\left(\widetilde{x_{p}}\right)\right), \tilde{d}\left(\tilde{y}_{\tau},(f, \varphi)\left(\widetilde{y_{\tau}}\right)\right), \tilde{d}\left(\tilde{x}_{p},(f, \varphi)\left(y_{\tau}{ }^{\sim}\right)\right)\right\} \\
& <\tilde{\eta}+\tilde{\gamma} \Rightarrow \tilde{d}\left((f, \varphi)\left(\tilde{x}_{p}\right),(f, \varphi)\left(y_{\tau}{ }^{\sim}\right)\right)<\tilde{\eta} .
\end{aligned}
$$

It is clear that the soft generalized Meir-Keeler contraction is a comparable soft Meir-Keeler contraction; we can easily conclude the following corollary.

Corollary 15. A soft generalized Meir-Keeler contraction on $(f, \varphi)$ which is a complete soft metric spaceMpossesses a fixed soft point.

\section{Data Availability}

No data is used!

\section{Conflicts of Interest}

The authors declare that they have no competing interests.

\section{Authors' Contributions}

Both authors contributed equally and significantly in writing this paper. Both authors read and approved the final manuscript.

\section{Acknowledgments}

This research was supported by the Ministry of Science and Technology of the Republic of China.

\section{References}

[1] L. A. Zadeh, "Fuzzy sets," Information and Control, vol. 8, pp. 103-112, 1965.

[2] D. A. Molodtsov, "Soft set theory-first results," Computers \& Mathematcs with Applications, vol. 37, no. 4-5, pp. 19-31, 1999.

[3] N. Cagman, S. Karatas, and S. Enginoglu, "Soft topology," Computers \& Mathematcs with Applications, vol. 61, pp. 1351-1358, 2011.

[4] S. Das and S. K. Samanta, "Soft metric," Annals of Fuzzy Mathematics and Informatics, vol. 6, no. 1, pp. 77-94, 2013.

[5] M. Abbas, G. Murtaza, and S. Romaguera, "Soft contraction theorem," Journal of Nonlinear and Convex Analysis, vol. 16, pp. 423-435, 2015.

[6] M. I. Ali, F. Feng, X. Liu, W. K. Min, and M. Shabir, "On some new operations in soft set theory," Computers \& Mathematcs with Applications, vol. 49, pp. 1547-1553, 2005.

[7] K. V. Babitha and J. J. Sunil, "Soft set relations and functions," Computers \& Mathematcs with Applications, vol. 60, no. 7, pp. 1840-1849, 2010.

[8] D. Chen, E. C. C. Tsang, D. S. Yeung, and X. Wang, "The parameterization reduction of soft sets and its applications," Computers \& Mathematcs with Applications, vol. 49, no. 5-6, pp. 757-763, 2005.

[9] C. G. Aras, A. Sonmez, and H. Cakall, "On soft mappings," 2013, https://arxiv.org/abs/1305.4545.

[10] S. Hussain and B. Ahmad, "Some properties of soft topological spaces," Computers \& Mathematcs with Applications, vol. 62, no. 11, pp. 4058-4067, 2011.

[11] P. K. Maji, A. R. Roy, and R. Biswas, "An application of soft sets in a decision making problem," Computers \& Mathematcs with Applications, vol. 44, no. 8-9, pp. 1077-1083, 2002.

[12] P. K. Maji, R. Biswas, and A. R. Roy, "Soft set theory," Computers \& Mathematcs with Applications, vol. 45, no. 4-5, pp. 555-562, 2003.

[13] P. Majumdar and S. K. Samanta, "On soft mappings," Computers \& Mathematcs with Applications, vol. 60, no. 9, pp. 2666-2672, 2010.

[14] M. Shabir and M. Naz, "On soft topological spaces," Computers \& Mathematcs with Applications, vol. 61, no. 7, pp. 1786-1799, 2011.

[15] S. Das and S. K. Samanta, "Soft real sets, soft real numbers and their properties," Journal of Fuzzy Mathematics, vol. 20, no. 3, pp. 551-576, 2012.

[16] C.-M. Chen, "Common fixed-point theorems in complete generalized metric spaces," Journal of Applied Mathematics, vol. 2012, Article ID 945915, 14 pages, 2012. 
[17] A. Meir and E. Keeler, "A theorem on contraction mappings," Journal of Mathematical Analysis and Applications, vol. 28, no. 2, pp. 326-329, 1969.

[18] I. Altuntas and K. Taşköprü, "Compactness of soft cone metric space and fixed point theorems related to diametrically contractive mapping," Turkish Journal of Mathematics, vol. 44, pp. 2199-2216, 2020.

[19] N. Çağman, F. Çıtak, and S. Enginoğlu, "Fuzzy parameterized fuzzy soft set theory and its applications," Turkish Journal of Fuzzy System, vol. 1, no. 1, pp. 21-35, 2010.

[20] F. Feng, Y. B. Jun, and X. Zhao, "Soft semirings," Computers \& Mathematcs with Applications, vol. 56, no. 10, pp. 2621-2628, 2008.

[21] G. Dymek and A. Walendziak, "Soft set theory applied to general algebras," Demonstratio Mathematica, vol. 48, no. 1, 2015.

[22] Q. H. Hamko, N. K. Ahmed, and A. B. Khalaf, "On soft pc-separation axioms," Demonstratio Mathematica, vol. 53, no. 1, pp. 67-79, 2020.

[23] T. M. Al-shami and M. E. El-Shafei, "Two new forms of ordered soft separation axioms," Demonstratio Mathematica, vol. 53, no. 1, pp. 8-26, 2020.

[24] T. M. Al-shami and M. E. El-Shafei, "Two types of separation axioms on supra soft topological spaces," Demonstratio Mathematica, vol. 52, no. 1, pp. 147-165, 2019. 